\title{
Eigenfunction convergence of the Rayleigh-Ritz-Meirovitch method and the FEM
}

\author{
C.A. Morales* and R. Goncalves \\ Departamento de Mecánica, Universidad Simón Bolivar, Apdo. 89000, Caracas 1080 A, Venezuela
}

Received 6 December 2004

Revised 3 October 2006

\begin{abstract}
Eigenfunction convergence characteristics of the finite element method and the Rayleigh-Ritz method with quasicomparison functions (RRMM) are compared. The RRMM has previously proved to be superior in comparing eigenvalue convergence characteristics. The importance of studying natural mode convergence is associated to the fact that the general response of a structure is a linear combination of these characteristic functions; in other words, accurate structural response attainment demands accurate structural modes in the analysis. It is shown that in this case the RRMM also produces superior results. A refined definition of quasicomparison functions is advanced.
\end{abstract}

\section{Introduction}

Vibration problems not admitting closed-form eigensolutions are by far the rule rather than the exception. In approximate methods to solve eigenproblems of distributed structures, attaining accurate eigenfunctions is as critical as obtaining accurate eigenvalues. The latter are squares of natural frequencies which are academically the only frequencies with which synchronous motion is possible and practically the frequencies of harmonic forces that generate resonance in the structural response. The former are the distributed amplitude of these synchronic motions and, more importantly, a function-space basis for the structural response to any excitation; more precisely, the expansion theorem establishes that the general response of a continuous system can be written as a linear combination of the system eigenfunctions [3]. Synchronous motion is a free and undamped vibration in which every point of the system executes harmonic motion with the same frequency, and no phase angle; coincidentally, the only initial conditions that imply this natural motion are an initial deformation as an eigenfunction and zero initial velocities. Again, this concept is of educational interest only; in nonclassroom structural systems the eigensolution cannot be attained in closed form and the actual interest is in that the numerical methods yield an accurate eigensolution so that precise natural frequencies and structural response analyses be possible.

An important point regarding eigensolution convergence is the fact that approximate or Ritz eigenvalues converge to actual eigenvalues faster than Ritz eigenfunctions to actual eigenfunctions; mathematically, according to Rayleigh-Ritz theory, Ritz eigenvalues are one order of magnitude more accurate than Ritz eigenfunctions [3]. The implication, perhaps overlooked, is that eigenfunction convergence should control the stop of numerical iterations rather than eigenvalue convergence. This in turn implies that more efficient approximate methods are to be preferred because computationally inferior techniques demand solutions of large-order discrete eigenvalue problems to satisfy aspecified eigenfunction accuracy, with the associated numerical and computational-cost problems. In this regard, the

${ }^{*}$ Corresponding author. CCS 91270, P.O. Box 025323, Miami, FL 33102-5323, USA. Tel.: +1 58212906 4075; Fax: +1 582129064062 ; E-mail: cmorales@usb.ve. 


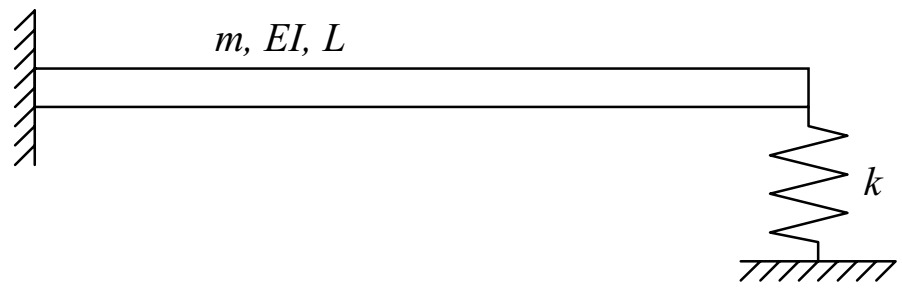

Fig. 1. Distributed structure.

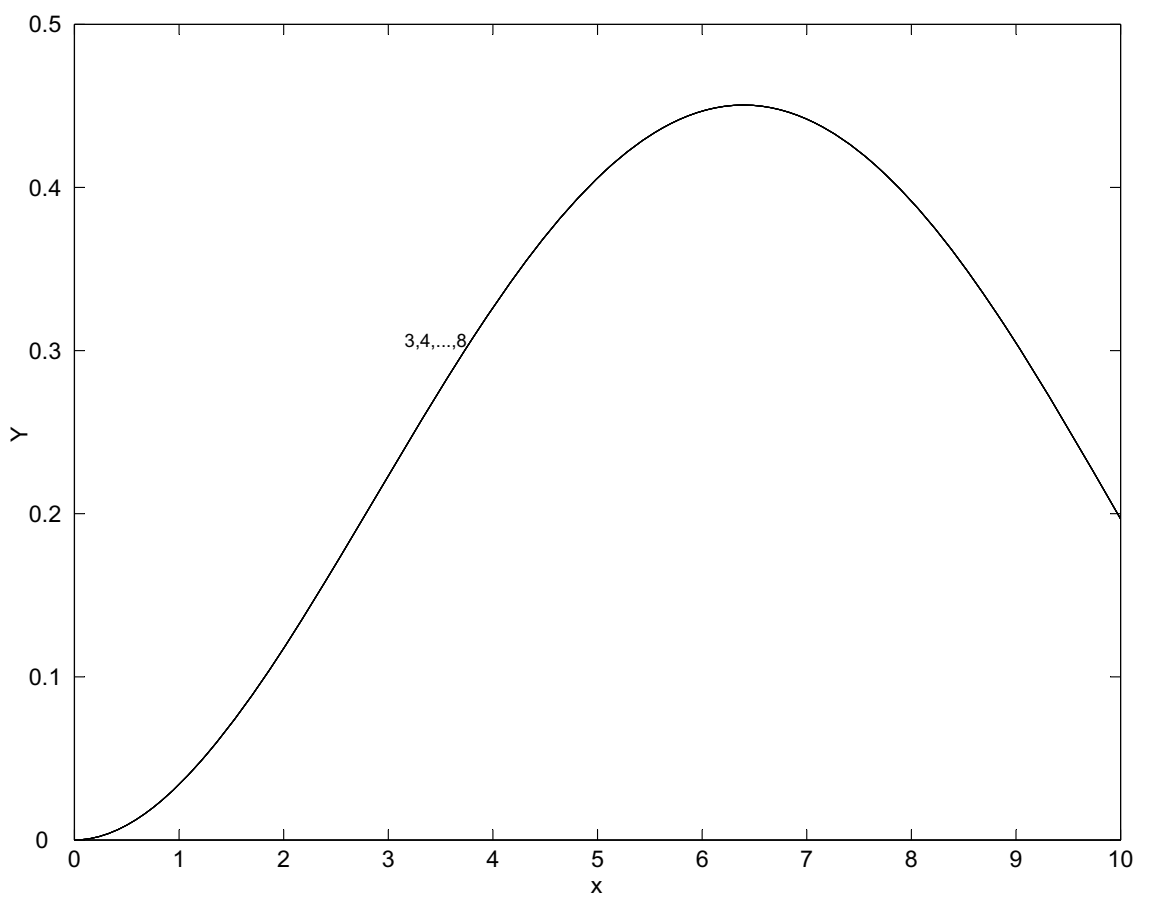

Fig. 2. RRMM first eigenfunction convergence $(3,4, \ldots, 8$ DOF).

Rayleigh-Ritz method with Meirovitch's quasicomparison functions has proved to be superior to the finite element method in eigenvalue convergence [4-6], when applicable.

In the classical Rayleigh-Ritz method [3], the use of mere admissible functions, commonly pertaining to the same family, can be characterised by poor or slow eigenvalue convergence, which in turn demands models with large numbers of degrees of freedom to try to achieve it; eigenfunction converge is basically out of question. This flaw in the method was noticed by Meirovitch and Kwak while studying eigenvalue convergence [4], and was explained by the fact that a finite linear combination of admissible functions of the same class is not able to satisfy natural boundary conditions. To solve this problem, a new class of functions was conceived by the same authors [4], the class of quasicomparison functions (QCF) defined by Meirovitch as "linear combinations of admissible functions capable of approximating the differential equation and the natural boundary conditions to any degree of accuracy by merely increasing the number $\mathrm{n}$ of terms in the approximating solution" [3]. The name comes from the fact that these combinations tend to act like comparison functions; for this to be true, the admissible functions must be from diverse families. Recall that admissible functions satisfy geometric boundary conditions; on the other hand, comparison functions must satisfy additionally the natural boundary conditions; thus, these are hard or impossible to obtain. To be precise and fair with the successful computational contribution, we call this Rayleigh-Ritz method with QCF, the Rayleigh-Ritz-Meirovitch method. The idea has been extended to analyze multibody structures such as frames; the superiority of eigenvalue-convergence characteristics of the methodology, when it is compared with the finite element method (FEM), is omnipresent [5-7]. 


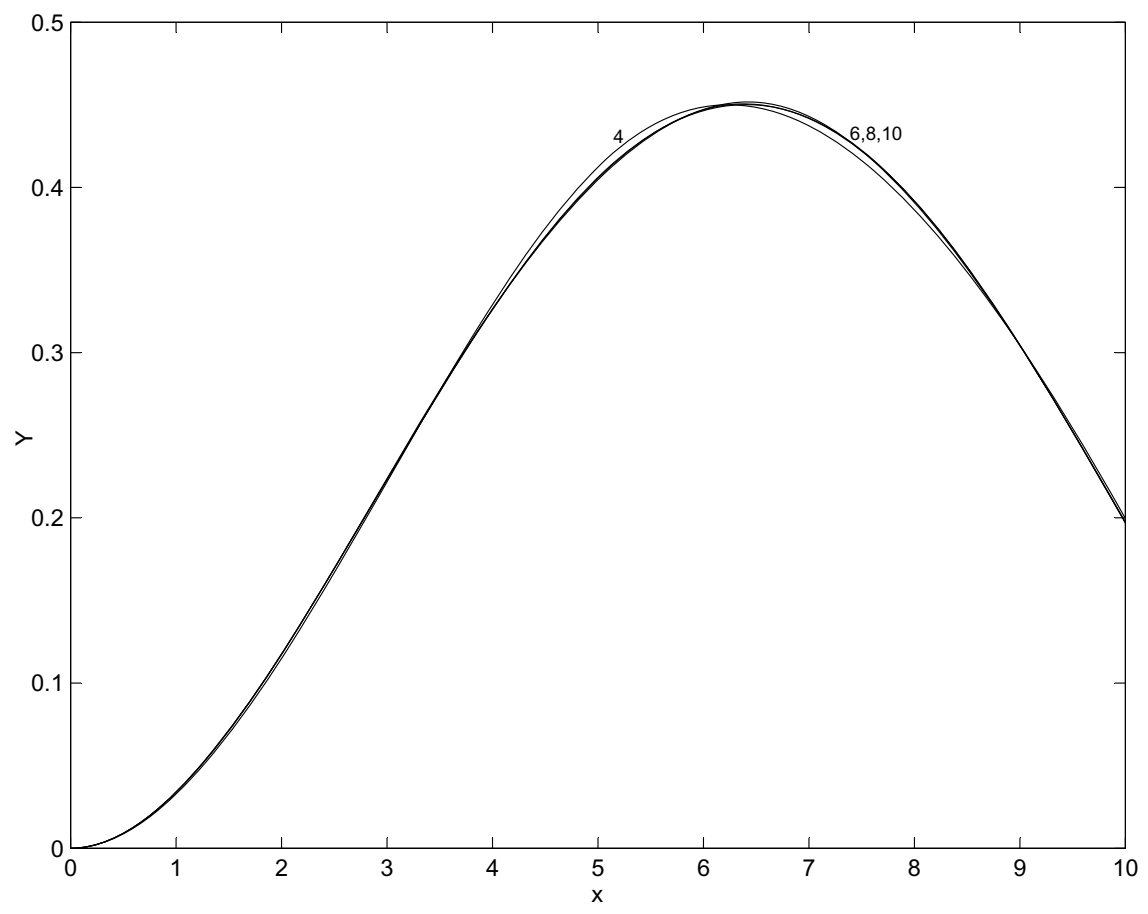

Fig. 3. FEM first eigenfunction convergence (4, 6, 8, 10 DOF).

In this investigation, eigenfunction convergence characteristics of the Rayleigh-Ritz-Meirovitch method (RRMM) and the conventional FEM are compared through the analysis of a simple structure. Moreover, the theoretical fact that Ritz eigenvalues are one order of magnitude more accurate than Ritz eigenfunctions is verified in practice. Similar results are, of course, not available in the literature. Furthermore, a refined definition of quasicomparison functions is advanced and a clearer definition of complementary boundary conditions is presented.

\section{The RRMM and refined QCF definition}

The Rayleigh-Ritz method with quasicomparison functions is applied to a simple structure to study eigenfunction convergence. A modest structure is analyzed because the interest is in that the essence of what is studied stands out; as a matter of fact, this Rayleigh-Ritz-Meirovitch method (RRMM) can be successfully applied to more complex structures [7]. The distributed system is depicted in Fig. 1; it is a beam of linear density $m$ and length $L$.

The differential eigenvalue problem associated with this uniform structure is defined by the equation

$$
E I \frac{\mathrm{d}^{4} Y}{\mathrm{~d} x^{4}}=\lambda m Y
$$

and the boundary conditions

$$
Y(0)=0 \quad \frac{\mathrm{d} Y}{\mathrm{~d} x}(0)=0 \quad \frac{\mathrm{d}^{2} Y}{\mathrm{~d} x^{2}}(L)=0 \quad E I \frac{\mathrm{d}^{3} Y}{\mathrm{~d} x^{3}}(L)=k Y(L)
$$

The eigensolution is made up by a denumerable infinite set of eigenvalues $\lambda_{i}$ and associated eigenfunctions $Y_{i}$. Recall that the order of a problem, $2 p$, defines absolutely the number of boundary conditions, $p$, at every point of a boundary.

Multiplication of Eq. (1) by $Y$ and integration over the domain yield 


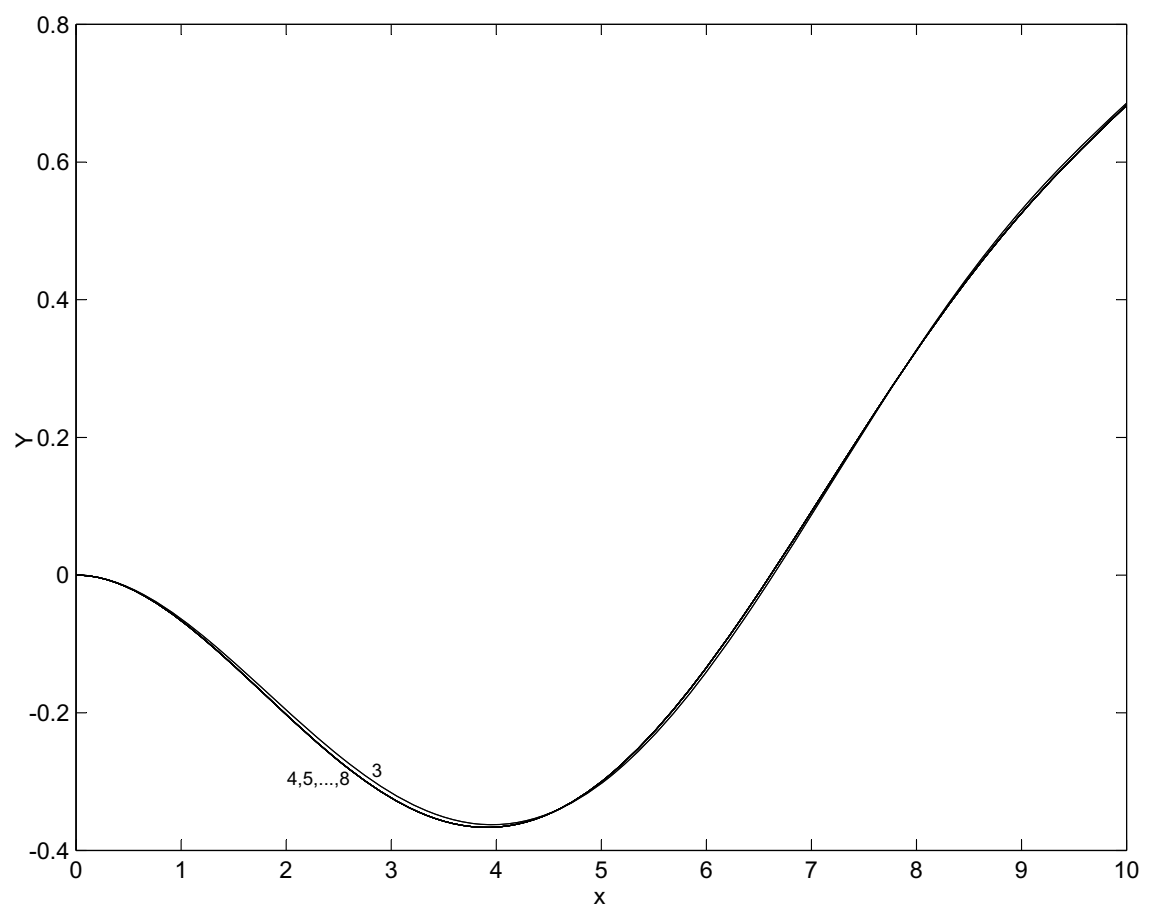

Fig. 4. RRMM second eigenfunction convergence $(3,4, \ldots, 8$ DOF).

$$
E I \int_{0}^{L} Y \frac{\mathrm{d}^{4} Y}{\mathrm{~d} x^{4}} \mathrm{~d} x=\lambda m \int_{0}^{L} Y^{2} \mathrm{~d} x
$$

which defines the Rayleigh's quotient [3] for this structure as

$$
R(Y)=\frac{E I \int_{0}^{L} Y \frac{\mathrm{d}^{4} Y}{\mathrm{~d} x^{4}} \mathrm{~d} x}{m \int_{0}^{L} Y^{2} \mathrm{~d} x}=\frac{E I \int_{0}^{L}\left(\frac{\mathrm{d}^{2} Y}{\mathrm{~d} x^{2}}\right)^{2} \mathrm{~d} x+k Y^{2}(L)}{m \int_{0}^{L} Y^{2} \mathrm{~d} x}
$$

where integration by parts was performed twice in the numerator which in this last form is an energy inner product; the denominator is the inner product of $m^{1 / 2} Y$ with itself.

The Rayleigh-Ritz method is based on the fact that Rayleigh's quotient has stationary values at the system eigenfunctions; indeed, the stationary values are the system eigenvalues. The original Rayleigh-Ritz method consists of assuming successive approximate solutions in the form of a finite series, in this case

$$
Y(x)=\sum_{i=1}^{n} c_{i} f_{i}(x)=\mathbf{f}^{T} \mathbf{c}
$$

where $c_{i}$ are coefficients that are determined by rendering Rayleigh quotient stationary and $f_{i}(x)$ are merely linearly independent admissible functions; the succession materialises as $n$ is varied. In the RRMM, admissible functions $f_{i}$ must satisfy certain conditions for $Y$ to qualify as a quasicomparison function (QCF). Indeed, as indicated in the Introduction, the vector $\mathbf{f}$ must include functions from different families; in fact, the current definition of QCF is in the same paragraph. Nonetheless, a refined and practical definition of QCF is advanced in this work.

A quasicomparison function is defined herein as a linear combination of admissible functions that is capable of satisfying the complementary boundary conditions. This definition differs from the original one in that, first, the requisite that the combination be able to approximate the differential equation to any degree of accuracy has been 


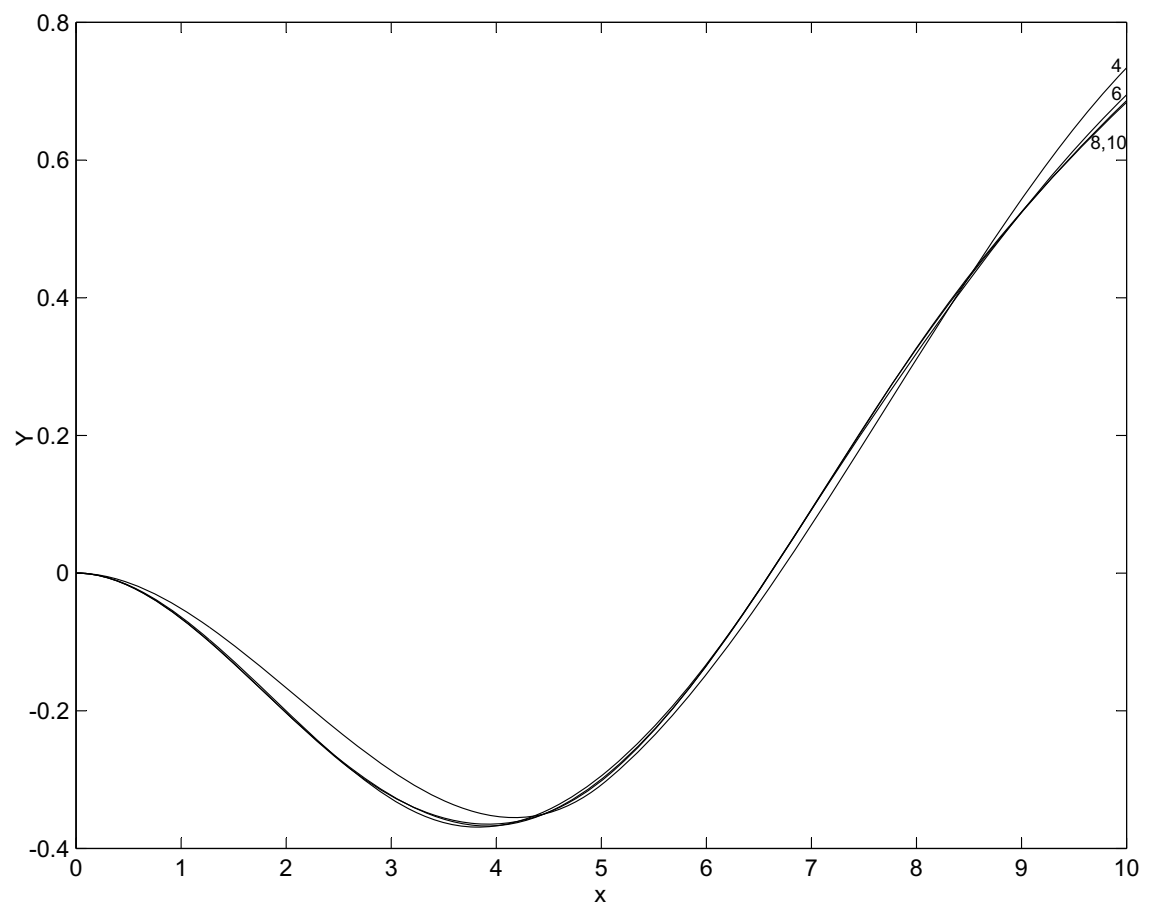

Fig. 5. FEM second eigenfunction convergence (4, 6, 8, 10 DOF).

dropped because in practice this cannot be guaranteed before hand; this can be assured only after the computational method, which demands a practical understanding of the QCF concept, has been implemented. More importantly, the focus has been shifted to complementary boundary conditions (CBC). The reason is that in most problems certain edge conditions arise that are not strictly boundary conditions, and the fact is that capability of satisfying these complementary conditions, which is itself advantageous, ensures also approximation of the natural boundary conditions, which was the original and sole concern [3]. This fact has been noted in all sample structures of reported investigations [4-7,9] and is clarified herein in the function-selection process for the sample structure. The term complementary boundary condition was introduced by Baruh and Tadikonda [1]. A clearer definition of the term in the case of beam systems (as the example or frames) is proposed in this work: edge condition that is expressed by an inequality of the type

$$
\left.\frac{\mathrm{d}^{n} Y}{\mathrm{~d} x^{n}}\right|_{\text {edge }} \neq 0
$$

where $n$ is up to $2 p-1,3$ in beam systems. CBC appear in both, multibody systems (substructure synthesis method) [5-7,9] and in the single-body case (classical Rayleigh-Ritz method) $[1,4]$.

The attention is turned back to the application of the RRMM to the sample structure; the interest is in explaining how a QCF is engineered, in this case $Y$ (Eq. (5)). Firstly, admissible functions $f_{i}$ as conventional Euler-Bernoulli beam natural functions represent an insuperable alternative because these are dynamically the most related to the vibration problem at hand; further, the characteristic twisting of higher eigenfunctions is automatically taken into account with this type of families. Next, the functions $f_{i}$, being admissible, must satisfy the two boundary conditions at $x=0$ because these are of the geometric class (Eqs (2a) and (2b)). Therefore, each family forming the QCF must be characterised by a clamped left end; note that the other conventional beam end configurations: free, pinned and sliding, do not satisfy both geometric boundary conditions. At the other end there are no geometric boundary conditions to be satisfied. Now, the QCF concept requires at least two families of functions; more importantly, the linear combination of these must be capable of satisfying the $\mathrm{CBC}$, which in this case can be expressed as

$$
\left.\frac{\mathrm{d}^{2} Y}{\mathrm{~d} x^{2}}\right|_{0} \neq\left. 0 \quad \frac{\mathrm{d}^{3} Y}{\mathrm{~d} x^{3}}\right|_{0} \neq 0 \quad Y(L) \neq\left. 0 \quad \frac{\mathrm{d} Y}{\mathrm{~d} x}\right|_{L} \neq\left. 0 \quad \frac{\mathrm{d}^{3} Y}{\mathrm{~d} x^{3}}\right|_{L} \neq 0
$$


Table 1

Required conditions in QCF and satisfaction by conventional configurations

\begin{tabular}{ccccc}
\hline QCF at $x=L$ & Clamped & Pinned & Sliding & Free \\
\hline$Y \neq 0$ & $\times$ & $\times$ & $\sqrt{ }$ & $\sqrt{ }$ \\
$\frac{\mathrm{d} Y}{\mathrm{~d} x} \neq 0$ & $\times$ & $\sqrt{ }$ & $\times$ & $\sqrt{ }$ \\
$\frac{\mathrm{d}^{3} Y}{\mathrm{~d} x^{3}} \neq 0$ & $\sqrt{ }$ & $\sqrt{ }$ & $\times$ & $\times$ \\
\hline
\end{tabular}

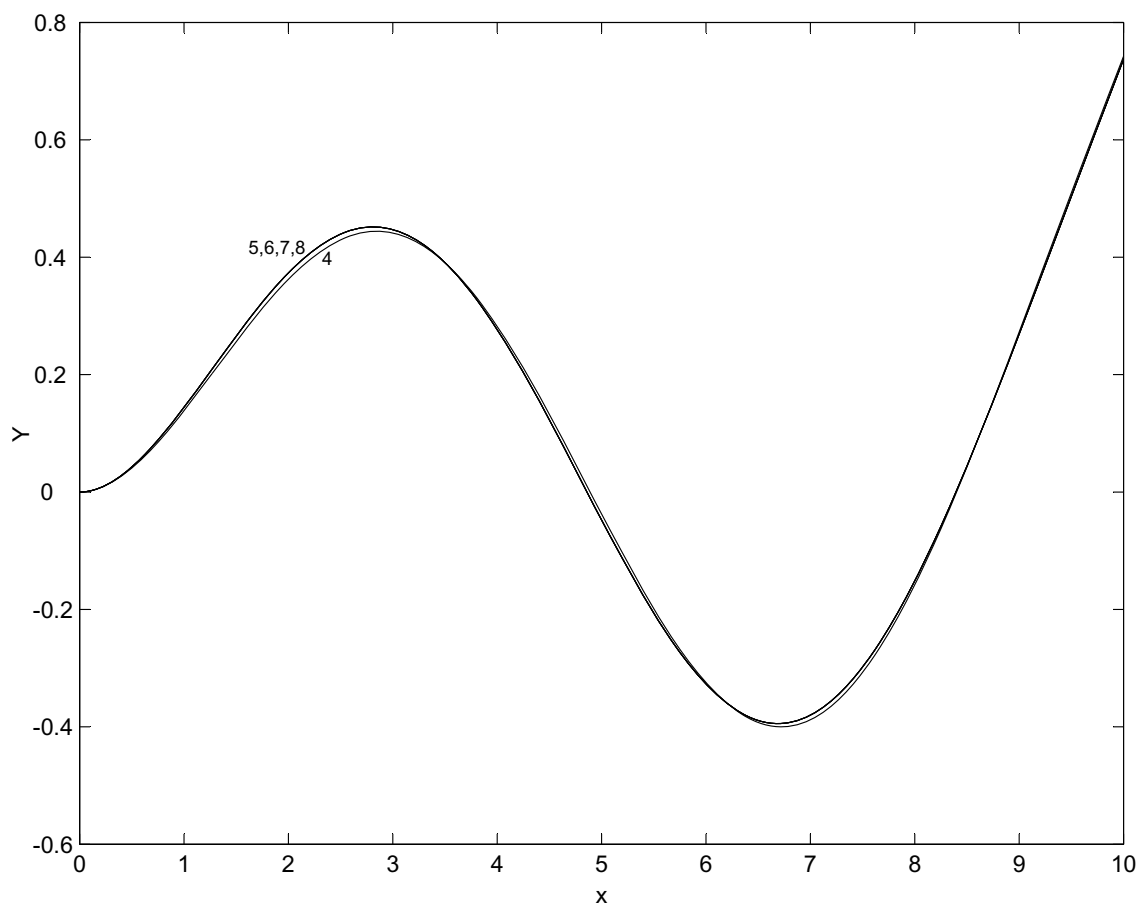

Fig. 6. RRMM third eigenfunction convergence $(4,5, \ldots, 8$ DOF).

Note that the first two conditions are already satisfied because it has been settled that the families be clamped at $x=0$; more importantly, notice that at the other end the combination must be able to allow for nonzero displacement, slope and shearing force. These desired capabilities in the QCF or linear combination at $x=L$ are shown in Table 1 where the satisfaction or not ( $\Pi$ or $\times$ ) of these conditions by the four conventional end configurations individually is also shown. It can be concluded that there is a superior 2-family mixture: clamped-free plus clamped-pinned, and it is selected. It is emphasised that the combination should have the freedom to satisfy the CBC; it is not that each function should satisfy all the CBC. Note that the other possibilities are clamped-free plus clampedclamped and clamped-sliding plus clamped-pinned. With any of the three possibilities, the approximation of the natural boundary conditions (NBC), Eq. (2c) and (2d), is guaranteed; as previously indicated, this fact, capability of satisfying $C B C$ guarantees approximation of $N B C$, has been manifested by reanalyzing all sample structures of reported investigations including multibody systems [4-7,9], and is the reason to base the QCF concept on the more general CBC rather than on NBC, which is proposed in this work. The refined and clearer definitions of QCF and $\mathrm{CBC}$, and the use of tools as Table 1 are expected to remove subjectivity and mystery from the function-selection process; in fact, it has been previously considered as "more of an art than a method" [3].

Thus, the QCF is formed by two families, clamped-free and clamped-pinned beam eigenfunctions,

$$
\begin{aligned}
& Y_{c f}=\cosh \beta_{1} x-\cos \beta_{1} x-\sigma_{1}\left(\sinh \beta_{1} x-\sin \beta_{1} x\right) \\
& Y_{c p}=\cosh \beta_{3} x-\cos \beta_{3} x-\sigma_{3}\left(\sinh \beta_{3} x-\sin \beta_{3} x\right)
\end{aligned}
$$




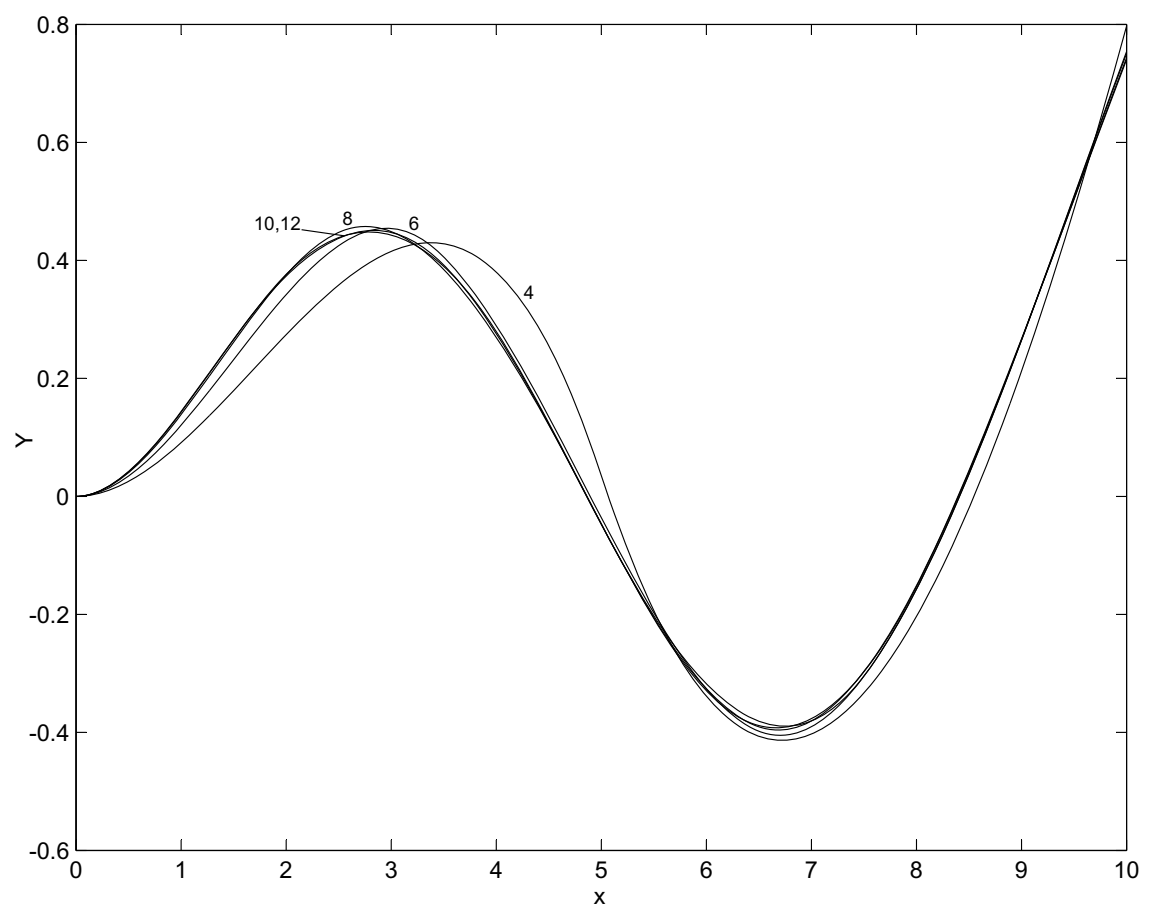

Fig. 7. FEM third eigenfunction convergence $(4,6, \ldots, 12$ DOF).

where the parameters $\beta$ and $\sigma$ are defined elsewhere [2,8]. Consequently, the admissible-function vector $\mathbf{f}$ in Eq. (5) is

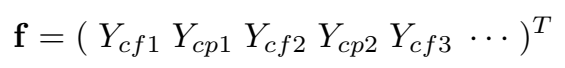

Next, Rayleigh quotient (Eq. (4)) can be written as

$$
R(Y)=\frac{k\left(\mathbf{f}^{T}(L) \mathbf{c}\right)^{2}+E I \int_{0}^{L}\left(\mathbf{f}^{\prime \prime T} \mathbf{c}\right)^{2} \mathrm{~d} x}{m \int_{0}^{L}\left(\mathbf{f}^{T} \mathbf{c}\right)^{2} \mathrm{~d} x}
$$

or

$$
R(Y)=\frac{\mathbf{c}^{T} \mathbf{K} \mathbf{c}}{\mathbf{c}^{T} \mathbf{M c}}
$$

Finally, according to Rayleigh-Ritz theory [3], rendering Rayleigh quotient stationary implies

$$
\mathbf{K c}=R \mathbf{M c}
$$

which is an algebraic eingenvalue problem; actually, it represents a sequence of algebraic eigenproblems approximating the authentic differential eigenvalue problem defined by Eqs (1) and (2). $\mathbf{M}$ and $\mathbf{K}$ are approximate mass and stiffness matrices, respectively, whose elements are defined as

$$
\begin{gathered}
m_{i j}=m \int_{0}^{L} f_{i} f_{j} \mathrm{~d} x \\
k_{i j}=k f_{i}(L) f_{j}(L)+E I \int_{0}^{L} f_{i}^{\prime \prime} f_{j}^{\prime \prime} \mathrm{d} x
\end{gathered}
$$




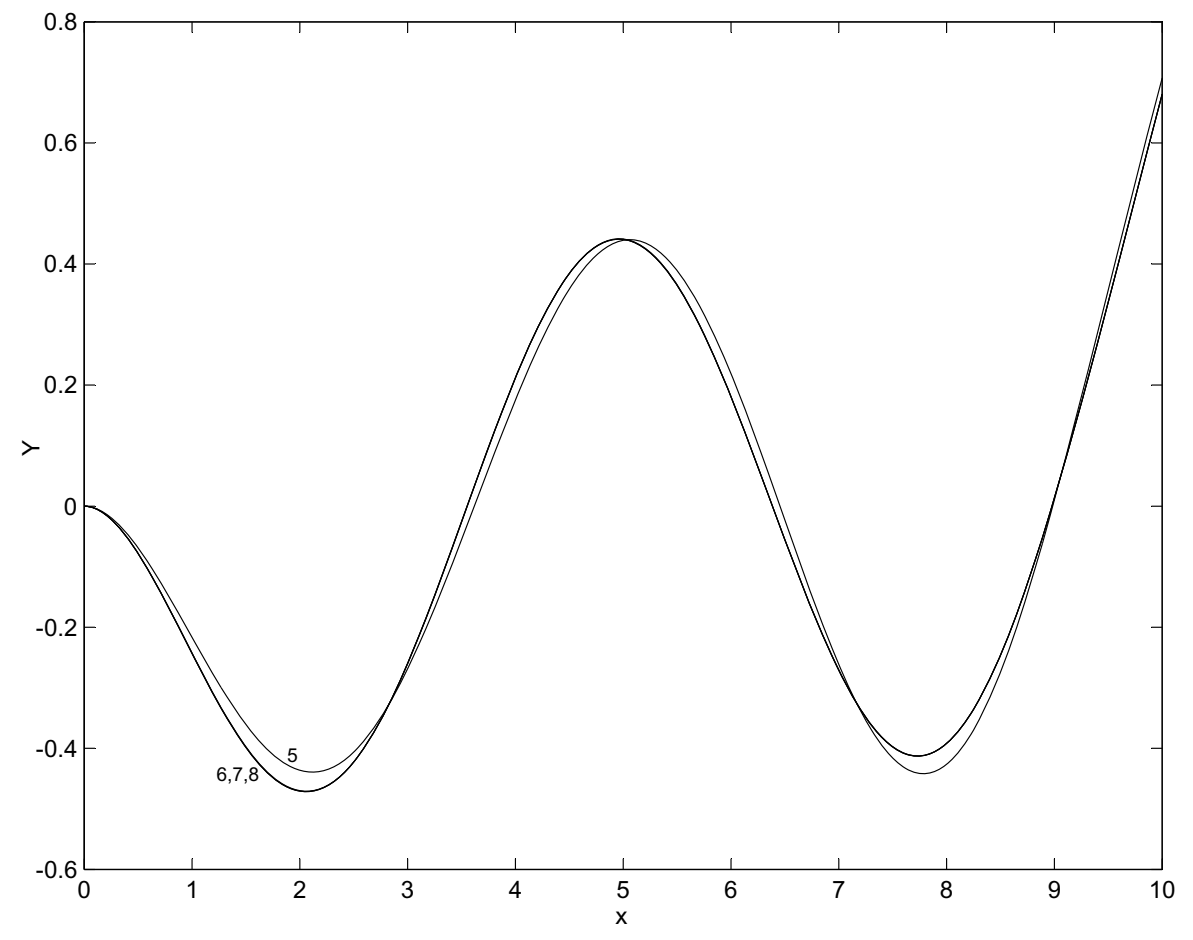

Fig. 8. RRMM fourth eigenfunction convergence (5, 6, 7, 8 DOF).

Of course, the solutions of the sequence of eigenproblems are a sequence of converging approximate eigenpairs; the approximate eigenfunctions are obtained by inserting the eigenvectors into the QCF (Eq. (5)). Closed-form integrals of products of trigonometric-hyperbolic functions may seem daunting; however, advantageous results, that are interestingly simple, are already available [2,8]. Last, the fact that a QCF may not be formed by completely independent functions is not a problem from a practical point of view as long as convergence is achieved before associated numerical impasses, such as almost-singular matrices, arise; if this is not the case, the solution is to add a third (fourth, and so on) family in the QCF.

\section{Results and comparison with the FEM}

The parameters of the structure in Fig. 1 are defined by assuming a steel $\left(\rho=7850 \mathrm{Kg} / \mathrm{m}^{3}, E=210^{11} \mathrm{~Pa}\right)$, $10 \mathrm{~m}$ long and square section $(0.1 \mathrm{~m})$ beam, and a spring stiffness $k=210^{5} \mathrm{~N} / \mathrm{m}$. The eigenvalue problem is solved by both, the RRMM and the FEM. The h-version FEM with Hermite cubics as interpolation functions is used. The convergence of the lowest four eigenvalues to six digits is shown in Table 2; eigenvalues are herein preferred over natural frequencies because it is an objective to verify the theoretical fact that Ritz eigenvalues are one order of magnitude more accurate than Ritz eigenfunctions. Results similar to those in Table 2 have been obtained before [4-7]; in fact, eigenvalue convergence is not the main matter of this investigation; nevertheless, it can be observed that, for a given accuracy, the solution yielded by the RRMM requires appreciably fewer degrees of freedom than the solution obtained by the FEM. To be specific, 6-digit convergence is achieved with 4, 5, 6 and 7 degrees of freedom (DOF) for the first, second, third and fourth eigenvalues, respectively, by means of the RRMM. In contrast, through the FEM that level of accuracy for barely the first eigenvalue is painfully achieved with 52 DOF; plainly, the third and fourth eigenvalues do not converge with 52 DOF. Of course, number of DOF is equivalent to order of matrices in the algebraic eigenproblems to be solved. The FEM first-eigenvalue convergence is so slow and poor that 6-digit convergence could have been called erroneously at 40 DOF as 392.029. As the theory predicts it, 
Table 2

Eigenvalue convergence

\begin{tabular}{|c|c|c|c|c|c|c|c|c|}
\hline \multirow[t]{2}{*}{ DOF } & \multicolumn{2}{|c|}{$\lambda_{1}$} & \multicolumn{2}{|c|}{$\lambda_{2}$} & \multicolumn{2}{|c|}{$\lambda_{3}$} & \multicolumn{2}{|c|}{$\lambda_{4}$} \\
\hline & RRMM & FEM & RRMM & FEM & RRMM & FEM & RRMM & FEM \\
\hline 3 & 392.029 & & 2319.83 & & & & & \\
\hline 4 & 392.028 & 398.212 & 2316.58 & 2437.95 & 9312.79 & 13451.0 & & \\
\hline 5 & 392.028 & & 2316.57 & & 9294.54 & & 32627.9 & \\
\hline 6 & 392.028 & 393.262 & 2316.57 & 2354.45 & 9294.53 & 9529.70 & 32134.7 & 43274.5 \\
\hline 7 & 392.028 & & 2316.57 & & 9294.53 & & 32134.1 & \\
\hline 8 & 392.028 & 392.420 & 2316.57 & 2329.32 & 9294.53 & 9458.94 & 32134.1 & 33038.2 \\
\hline 10 & & 392.189 & & 2321.93 & & 9371.05 & & 32910.9 \\
\hline 12 & & 392.106 & & 2319.19 & & 9333.55 & & 32561.9 \\
\hline 14 & & 392.070 & & 2317.99 & & 9316.25 & & 32379.3 \\
\hline 16 & & 392.053 & & 2317.41 & & 9307.52 & & 32282.9 \\
\hline 18 & & 392.044 & & 2317.09 & & 9302.74 & & 32229.1 \\
\hline 20 & & 392.038 & & 2316.91 & & 9299.97 & & 32197.4 \\
\hline 24 & & 392.033 & & 2316.73 & & 9297.19 & & 32165.2 \\
\hline 28 & & 392.031 & & 2316.66 & & 9295.98 & & 32151.1 \\
\hline 32 & & 392.030 & & 2316.62 & & 9295.38 & & 32144.1 \\
\hline 36 & & 392.029 & & 2316.60 & & 9295.07 & & 32140.4 \\
\hline 40 & & 392.029 & & 2316.59 & & 9294.88 & & 32138.2 \\
\hline 44 & & 392.029 & & 2316.58 & & 9294.77 & & 32136.9 \\
\hline 48 & & 392.029 & & 2316.58 & & 9294.70 & & 32136.1 \\
\hline 52 & & 392.028 & & 2316.57 & & 9294.66 & & 32135.5 \\
\hline
\end{tabular}

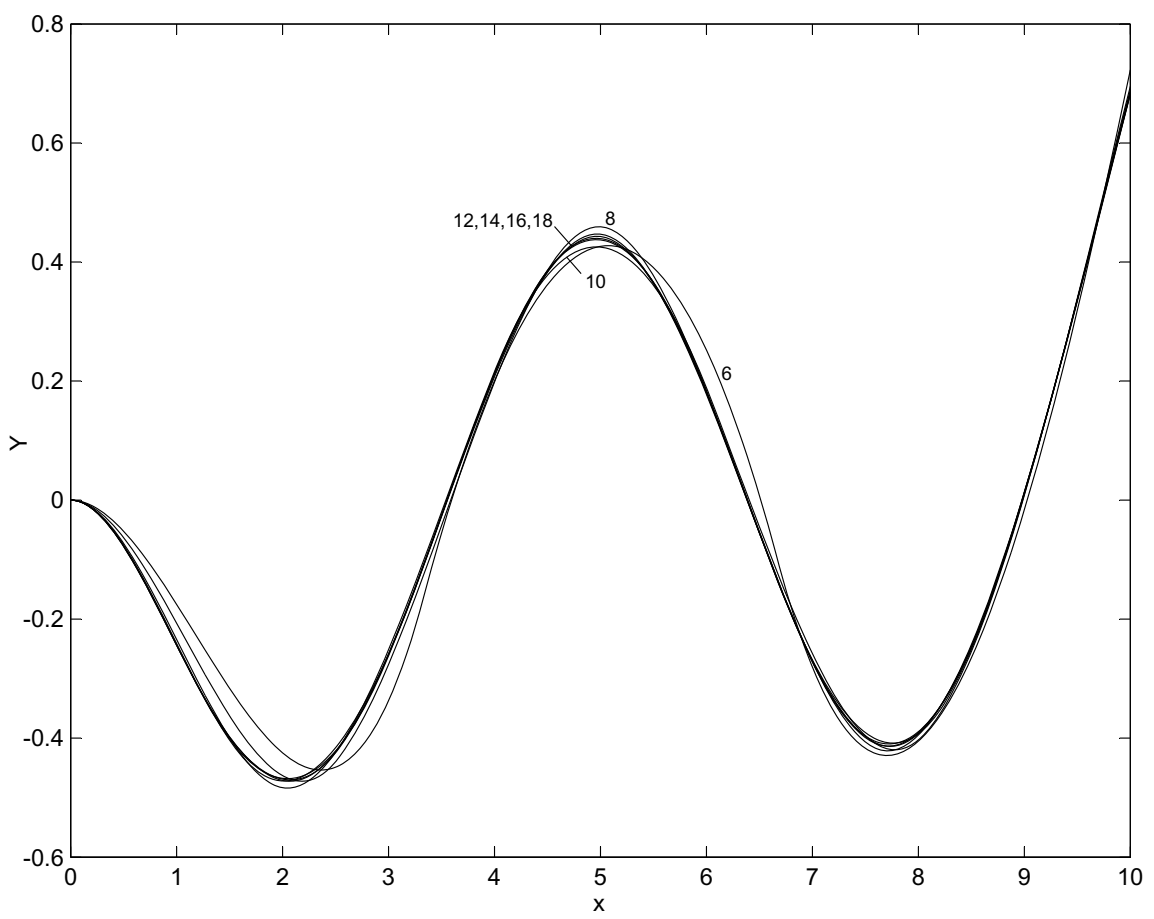

Fig. 9. FEM fourth eigenfunction convergence $(6,8, \ldots, 18$ DOF).

eigenvalue convergence of Rayleigh-Ritz based methods is uniform and from above; nonetheless, it can be added that the order of convergence rate of the RRMM is greater than that of the FEM.

The study of eigenfunction convergence, which is the subject of this investigation, demands the establishment of a normalisation procedure because an eigenfunction, either closed-form or approximate, possesses a definite shape but not a definite amplitude. Without normalised approximate eigenfunctions, it is not possible to evaluate convergence 
Table 3

Eigenfunction convergence at $x=L$

\begin{tabular}{|c|c|c|c|c|c|c|c|c|}
\hline \multirow[t]{2}{*}{ DOF } & \multicolumn{2}{|c|}{$Y_{1}(L)$} & \multicolumn{2}{|c|}{$Y_{2}(L)$} & \multicolumn{2}{|c|}{$Y_{3}(L)$} & \multicolumn{2}{|c|}{$Y_{4}(L)$} \\
\hline & RRMM & FEM & RRMM & FEM & RRMM & FEM & RRMM & FEM \\
\hline 3 & 0.196812 & & 0.685481 & & & & & \\
\hline 4 & 0.196813 & 0.199625 & 0.682420 & 0.734139 & 0.742279 & 0.797323 & & \\
\hline 5 & 0.196813 & & 0.682413 & & 0.737242 & & 0.707400 & \\
\hline 6 & 0.196813 & 0.197413 & 0.682413 & 0.694755 & 0.737241 & 0.753985 & 0.680748 & 0.722811 \\
\hline 7 & 0.196813 & & 0.682413 & & 0.737241 & & 0.680664 & \\
\hline 8 & 0.196813 & 0.197006 & 0.682413 & 0.686375 & 0.737241 & 0.750747 & 0.680664 & 0.682116 \\
\hline 10 & & 0.196893 & & 0.684046 & & 0.743430 & & 0.695213 \\
\hline 12 & & 0.196852 & & 0.683203 & & 0.740374 & & 0.689156 \\
\hline 14 & & 0.196834 & & 0.682841 & & 0.738979 & & 0.685639 \\
\hline 16 & & 0.196826 & & 0.682664 & & 0.738277 & & 0.683719 \\
\hline 18 & & 0.196821 & & 0.682570 & & 0.737895 & & 0.682629 \\
\hline 20 & & 0.196818 & & 0.682516 & & 0.737674 & & 0.681980 \\
\hline 24 & & 0.196816 & & 0.682463 & & 0.737452 & & 0.681315 \\
\hline 28 & & 0.196815 & & 0.682440 & & 0.737355 & & 0.681021 \\
\hline 32 & & 0.196814 & & 0.682429 & & 0.737308 & & 0.680875 \\
\hline 36 & & 0.196814 & & 0.682423 & & 0.737283 & & 0.680796 \\
\hline 40 & & 0.196814 & & 0.682419 & & 0.737269 & & 0.680751 \\
\hline 44 & & 0.196814 & & 0.682417 & & 0.737260 & & 0.680724 \\
\hline 48 & & 0.196813 & & 0.682416 & & 0.737254 & & 0.680706 \\
\hline
\end{tabular}

and to compare results of the RRMM and the FEM. Normalisation processes are arbitrary, so in our case it could be as simple as defining the value of the functions at $x=L$, for example $Y(L)=1$; nonetheless, in this case eigenfunction convergence would be apparently better at points close to $x=L$ than on the middle of the domain; in fact, at $x=L$ there would be no convergence at all. An ideal normalisation procedure does not privilege one point over the rest on the domain; thus, a process involving integration seems to be the answer. Now, a normalisation based on requiring simply that

$$
\int_{0}^{L} Y \mathrm{~d} x=1
$$

would not be correct, at least computationally, because only the first eigenfunction is not sign-changing; in fact, higher beam eigenfunctions tend to possess zero area. For this reason, a suitable normalisation procedure and the one used here is

$$
\int_{0}^{L} Y^{2} \mathrm{~d} x=1
$$

The convergence of the first eigenfunction is shown in Fig. 2 (RRMM) and Fig. 3 (FEM). As in the case of eigenvalue convergence, the RRMM involves appreciably fewer DOF than the FEM for eigenfunction convergence; in fact, the RRMM first eigenfunction convergence process is so outstanding that it cannot be viewed on the graph, i.e. the 6 approximate eigenfunctions are one over each other. In contrast, 8 DOF are required for FEM graphic convergence. The convergence of higher eigenfunctions is shown in Figs 4 to 9 . The superiority of the RRMM is omnipresent; to be precise, only 4, 5 and 6 DOF are demanded for graphic convergence of the second, third and fourth eigenfunctions, respectively; in contrast, 10, 12 and $18 \mathrm{DOF}$, respectively, are required for FEM convergence. Note that at any iteration the highest eigenpair is never presented because this result is meaningless.

The previous discussion is more qualitative than quantitative. In order to analyze quantitatively, Table 3 shows the convergence of the eigenfunctions at $x=L$, for example. Once again, for a given accuracy, the solution yielded by the RRMM requires appreciably fewer DOF than the solution produced by the FEM. Specifically, 6-digit convergence is achieved with 4, 5, 6 and 7 DOF for the first, second, third and fourth eigenfunctions, respectively, through the RRMM. In contrast, by the FEM that level of accuracy for merely the first eigenfunction is arduously attained with 48 DOF. The RRMM convergence is uniform and unidirectional, from below (first function) or above (second, third and 


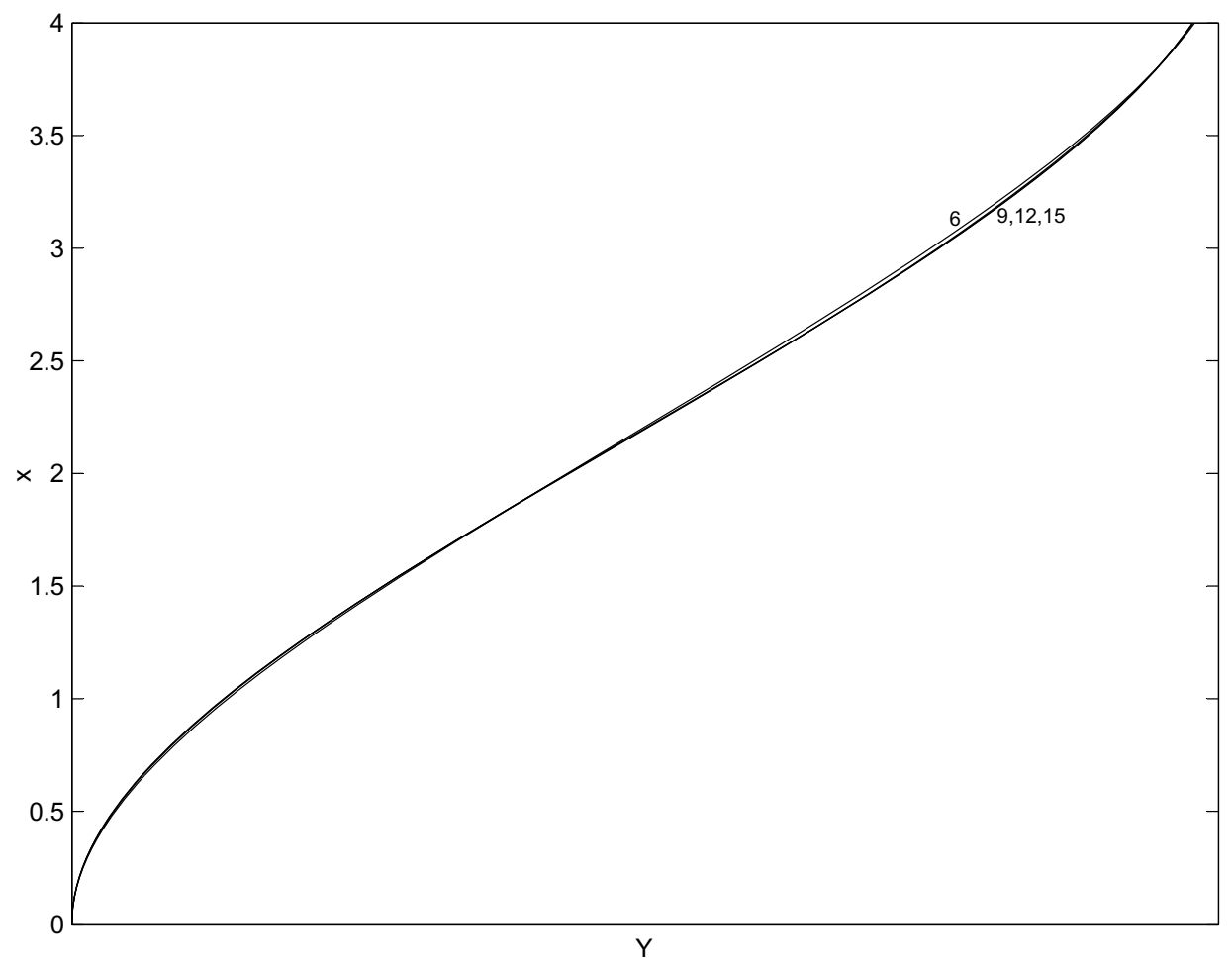

Fig. 10. Portal frame column RRMM first mode convergence $(6,9,12,15 \mathrm{DOF})$.

fourth); this is an important result that has not been proved theoretically. A theoretical result that this investigation can confirm is the fact that Ritz eigenvalues are one order of magnitude more accurate than Ritz eigenfunctions [3]. For example, at the level of $4 \mathrm{DOF}$, the second eigenvalue is 5-digit accurate and the corresponding function (at $x=L)$ is 4-digit accurate; however, the third eigenvalue and the corresponding function are both 2-digit accurate. There is no problem here as it is common in numerical or computational results to have a numerical theory not perfectly matched; further, rather than a theoretical result, the previous statement on accuracies of eigenpairs is solely an intuitive conclusion [3]. Finally, one reviewer required the analysis of a more practical structure; the portal frame in [6] has been chosen and analysed, considering a steel structure: $m_{c}=m_{b}=78.5 \mathrm{Kg} / \mathrm{m}, E I_{c}=E I_{b}=$ $1.666710^{6} \mathrm{~N} \mathrm{~m}^{2}, L=l=4 \mathrm{~m}$. As an example and because of space constraints, the convergence of the first mode (only the part corresponding to the left column) is shown in Fig. 10.

The engineering implication of the results is that in dynamic structural response analysis, both, low-order eigenproblems for mode attainment and final highly accurate response results can only be achieved through particularly efficient discretisation methods, as the RRMM. Both characteristics are not possible in response analysis by means of less effective methods as the FEM; in fact, none of these could be a possibility. Finally, another important advantage of the RRMM is that the general structural response can be written in a very simple form; for the sample structure the expression is simply

$$
y(x, t)=\sum_{j=1}^{N} Y_{j}(x) q_{j}(t)=\sum_{j=1}^{N} \mathbf{f}^{T} \mathbf{c}_{j} q_{j}
$$

where $N$ is the number of modes considered relevant in the response and $q_{j}$ are the generalised coordinates; of course, the order of eigenvector $\mathbf{c}_{j}$ is equal to the number of DOF required for convergence of the corresponding mode $Y_{j}$. 


\section{Conclusion}

Eigenfunction convergence characteristics of the finite element method and the Rayleigh-Ritz-Meirovitch method (RRMM) were compared. In addition, the theoretical fact that Ritz eigenvalues are one order of magnitude more accurate than Ritz eigenfunctions was verified in practice, a practical and refined definition of quasicomparison function was advanced, and a clearer definition of complementary boundary condition was presented. It was demonstrated that RRMM mode convergence characteristics are superior to those of the conventional FEM. Therefore, highly accurate response analyses based on solutions of low-order eigenproblems are amazingly possible.

\section{References}

[1] H. Baruh and S.S.K. Tadikonda, Another look at admissible functions, Journal of Sound and Vibration 132 (1989), $73-87$.

[2] R.D. Blevins, Formulas for natural frequency and mode shape, Van Nostrand Reinhold, USA, 1979.

[3] L. Meirovitch, Principles and techniques of vibrations, Prentice Hall, USA, 1997.

[4] L. Meirovitch and M.K. Kwak, Convergence of the classical Rayleigh Ritz method and the finite element method, AIAA Journal 28 (1990), 1509-1516.

[5] L. Meirovitch and M.K. Kwak, Rayleigh-Ritz based substructure synthesis for flexible multibody systems, AIAA Journal 29 (1991), 1709-1719.

[6] C.A. Morales, Rayleigh-Ritz based substructure synthesis for multiply supported structures, Journal of Vibration and Acoustics 122 (2000), 2-6.

[7] C.A. Morales, Dynamic analysis of frames by a Rayleigh-Ritz based substructure synthesis method, Engineering Structures 22 (2000), 1632-1640.

[8] C.A. Morales and J.F. Ramírez, Further simplest-expression integrals involving beam eigenfunctions and derivatives, Journal of Sound and Vibration 253 (2002), 518-522.

[9] J.F. Ramírez, Autosolución de problemas de pandeo y conducción de calor mediante síntesis subestructural, M.Sc. thesis, Universidad Simón Bolívar, Venezuela, 2002. 

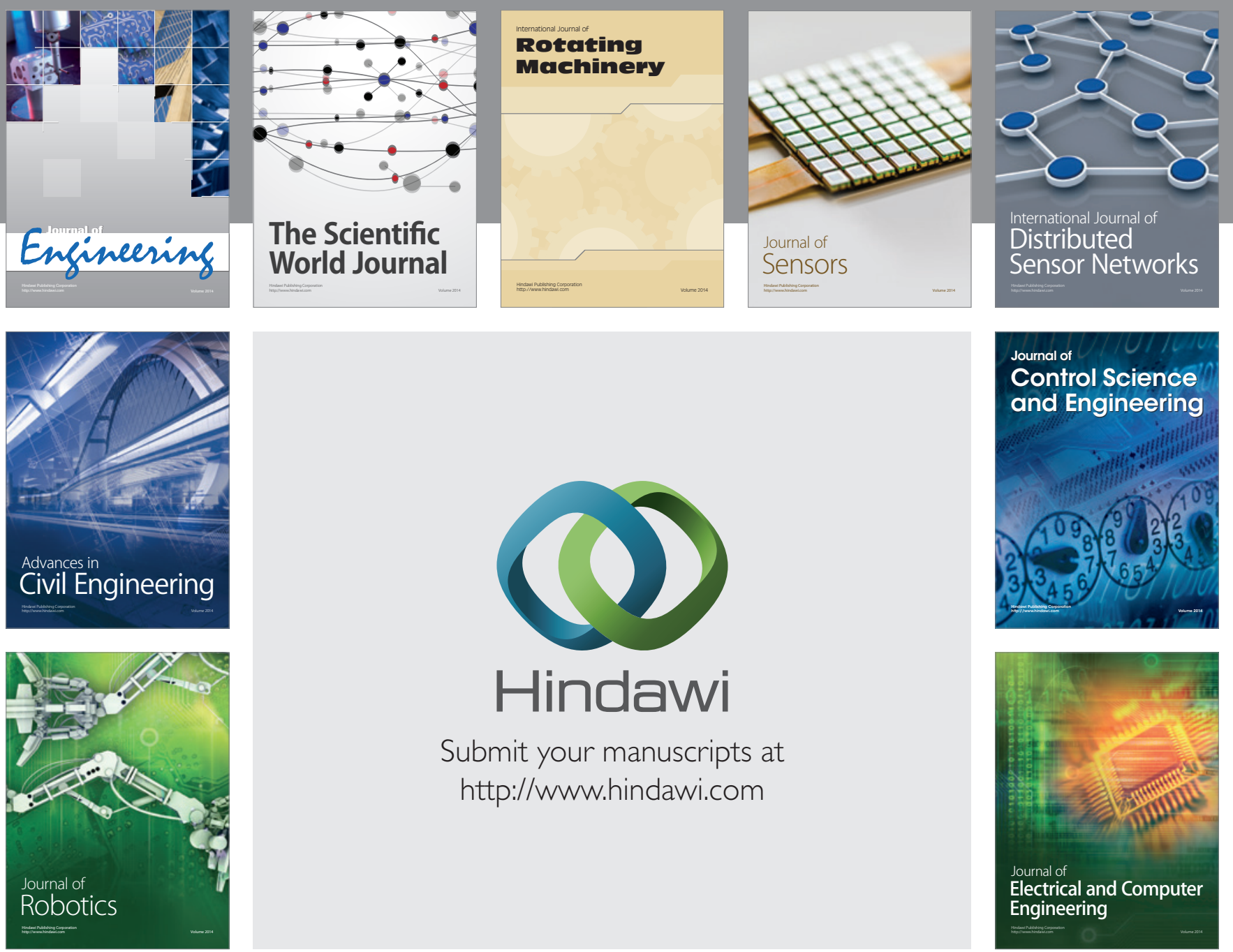

Submit your manuscripts at

http://www.hindawi.com
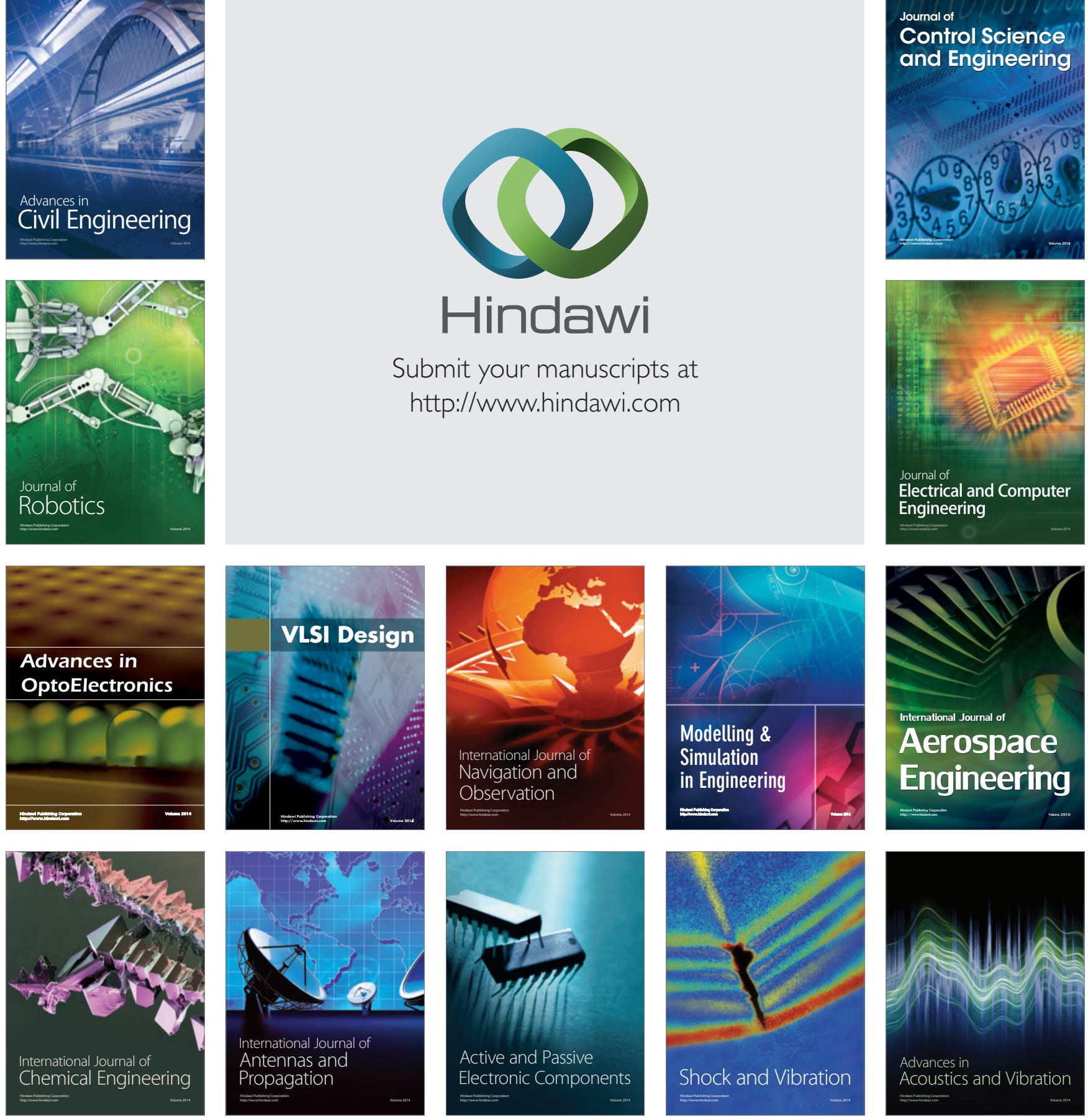